Excitation and Damping of Drift Waves

A. Y. Wong and R. Rowberg

November, 1966

P-8
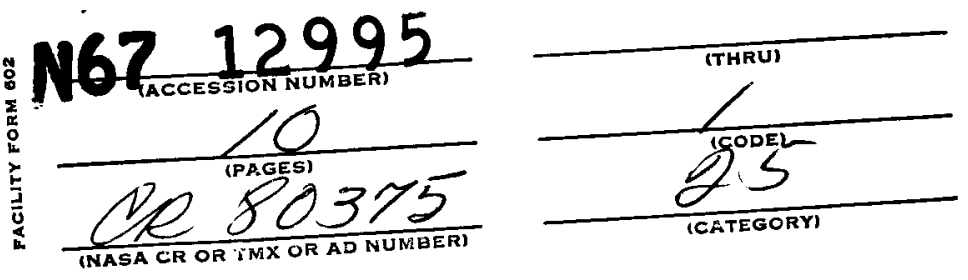

GPO PRICE

CFSTI PRICE(S) S

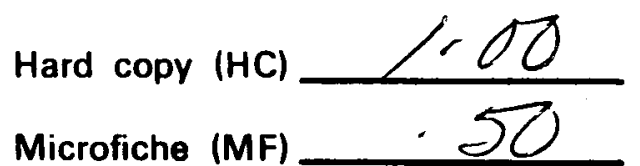

Plasma Physics Group

ff 653 July 65

Department of Physics

University of California

Los Angeles, California

This research was supported by the National Aeronautics and Space Administrationt under grant NGR 05-007-066 and funds from the Physics Department. 


\title{
Excitation and Damping of Drift Waves
}

\author{
A. Y. Wong and R. Rowberg \\ Department of Physics, University of California \\ Los Angeles, California
}

In an inhomogeneous plasma immersed in a magnetic field, there are waves associated with the density and temperature gradients. Such waves are electrostatic in nature and propagate in a direction perpendicular to both the magnetic field and the gradients. There have been extensive theoretical calculations ${ }^{1-3}$ and experimental observations ${ }^{4-9}$ regarding these waves in the unstable regime. However the damping of these waves in the stable regime has not been investigated. It is the purpose of this paper to report the excitation and damping of such drift waves in the stable regime in a highly ionized plasma. 10 The excitation of small amplitude waves $\left(\frac{e d}{\mathrm{kT}} \leq 0.1\right)$ permits one to verify the dispersion relation derived from a linearized theory as in the case of ion acoustic waves. 11

This experiment makes use of the characteristic of the drift wave that it is a standing wave in the axial direction and a propagating wave in the azimuthial direction. Thus after the wave is excited to steady state, the excitation signal is withdrawn and the temporal damping is measured. This measurement is meaningful because the wave does not propsrate away from the observation region. The experiment is conducted on a $\mathrm{Q}$-device ${ }^{12}$ which produces a potassium plasma confined axially by a hot tungsten plate at one end and a cold tantalum plate at the other. 
The plasma column length is adjustable between $100 \mathrm{~cm}$ and $65 \mathrm{~cm}$ by moving, either end. Radially, the plasma is confined by an axial magnetic field and has a diameter of $2.5 \mathrm{~cm}$. The experiment was performed under suitable conditions $\left(10^{10}<\mathrm{n}<7 \times 10^{10}\right.$ and $1.2 \mathrm{KW} \leq \mathrm{Plate}<1.5 \mathrm{KW}$ and sufficiently high magnetic field) such that the hot end plate is electron rich and the naturally occurring background fluctuations have strength $\frac{\mathrm{e} \phi}{\mathrm{KT}} \leq .01$. The drift wave was excited by inserting a floating $2 \mathrm{~cm} \times 2 \mathrm{~cm}$ tungsten grid $(.0025 \mathrm{~cm}$ diameter wire and $.05 \mathrm{~cm}$ spacing) into the plasma near the maximum density gradient, the grid plane being parallel to the axial direction. Figure 1 shows a typical excited wave and its temporal damping when the excited signal is withdrawn. Phase measurements with Langmuir probes show that the wave travels azimuthally in the direction of the electron diamagnetic drift and exhibits an $m=1$ characteristic. This wave reverses its direction upon reversal of the magnetic field. The optimium frequency of excitation indeed corresponds to the naturally excited frequency in the unstable regime as density or end-plate sheath conditions are changed.

Under our experimental conditions in which $k_{z} \lambda_{m f p} \ll 1, \omega \tau_{e i} \ll 1$, and $\omega \tau_{e e} \ll 1$, where $k_{z}$ is the wave number along $\vec{B}, \lambda_{m f p}$ is the electron or ion mean free path, $\omega$ is the excitation frequency, $\tau_{e i}$ is the electronion collision time and $\tau_{e e}$ is the electron-electron collision time, we have employed fluid equations for both ions and the electrons in the manner of Moiseev and Sagdeev ${ }^{1}$ and Chen $^{2}$ and have derived the following expression for the destabilizing term for $k_{L} R_{L}<1$

$$
\omega_{g}=+\left(2-\frac{1}{r}\right) v_{e i} \frac{m_{e}}{M}(m)^{4}\left(k_{\perp} R_{L}\right)^{4}\left(\frac{1}{n_{o}} \frac{\partial n_{o}}{\partial r}\right)^{2} \frac{1}{k_{z}^{2}}
$$


where

$$
\begin{array}{ll}
v_{e i}=\text { electron ion collision frequency } & k_{2}, k_{1}=\underset{\text { wave number resp. }}{\text { axial, azimuthal }} \\
m=\text { azimuthal mode number } & R_{L}=\text { ion cyclotron radius } \\
m_{e}, N=\text { electron, ion mass respectively } & \gamma=\text { ratio of specific heats }
\end{array}
$$

In the stable regime this destabilizing tor? $\omega_{g}$ is smaller than the damping term $\omega_{0}$ which most probably arises from losses due to reflection from the end plates. We have chosen as experimental parameters the axial magnetic field $B$ and the colum length $L$ which changes $\omega_{g}\left(\alpha \frac{L^{2}}{B}\right)$ appreciably but leaves $\omega_{0}$ relatively unaffected. Thus the measured overall damping rate $\left(\omega_{0}-\omega_{g}\right)$ varies principally with $\omega_{g}$. Figures $2 a$ and $2 b$ show the variation of damping rate as function of $B$ and $L .13$

The discrepancy in Fig. $2 a$ is increasingly large at lower magnetic field where $k_{L} R_{L} \rightarrow 0.3$. In fact during certain runs we have seen the overall damping first decrease as $B$ is lowered then increase again when $B$ is lowered further beyond 1400-1700 gauss. The discrepancy can be attributed to the fact that large Larmor radius effects have not been included in the present theory. ${ }^{14}$ The variation of damping with column length in Fig. $2 b$ seems to indicate that resistive effects are important. Collisionless damping would have required a linear dependence on L. 6 We have also observed that the damping decreases with increasing density and decreasing end-plate power which controls the plasma temperature. Since $v_{e i}-n / T^{3 / 2}$ this fact qualitatively agrees with the theoretical prediction of $\omega_{g} * v_{e i} \cdot$ Finally it is observed that the damping rate varies with the excitation amplitude when $\frac{e \phi}{\mathrm{KT}} \geq 20 \%$. The damping rate at $\frac{\mathrm{e} \mathcal{K}}{\mathrm{KT}} \approx 30 \%$ is in fact twice as large as that at $\frac{\mathrm{e} \phi}{\mathrm{KT}} \approx 10 \%$. This 
experimental fact points out that one must be careful in using a linearized theory to interpret quasilinear or nonlinear effects of drift waves.

In conclusion, we have demonstrated the excitation of drift waves whose damping can be explained partially by a fluid theory including resistivity. This present method of excitation promises a quantitative differentiation between collisionless and resistive damping and an investigation of the transition from linear to quasilinear regimes.

\section{Acknow ledgements}

We are grateful to Dr. P. Schram for many useful discussions. The able help of Mir. Zalton Lucky and the UCLA plasma group is gratefully acknowledged. This research was supported in part by the National Aeronautics and Space Administration under grant NGR 05-007-066 and by funds from the UCLA Physics Department. 
1. S. S. Moissev and R. Z. Sagdeev, Zh. Tekhn. Fiz. 34, 248 (1964), (English Trans1.: Soviet Phys. - Tech. Phys. 9, 196 (1964).

2. F. F. Chen, Princeton University Plasma Physics Laboratory Report, MATT-313 (1964); Phys. Fluids $\underline{8}, 1323$ (1965); Phys. Fluids 9, 965 (1966).

3. J. D. Jukes, Phys. Fluids 7, 1468 (1964).

4. N. D'Angelo and R. W. Motley, Phys. Fluids 6, 422 (1963).

5. H. Lashinsky, Phys. Rev. Letters 12, 121 (1964); 13, 47 (1964).

6. N. S. Buchel'nikova, Nucl. Fusion 4 , 165 (1964).

7. C. W. Hartman and R. H. Munge1, UCRL Report (1965).

8. J. A. Decker, Jr., Sperry Rand Research Center Report SRRC-RR-66-6 (1966).

9. L. Enriques, Princeton University Plasma Physics Laboratory Report, MATT-408 (1965) .

10. A. Y. Wong and R. Rowberg, UCLA Plasma Physics Group Report R-8, July (1966) .

11. A. Y. Wong, R. Motley and N. D'Ange1o, Phys. Rev. 133, A436 (1964).

12: N. Rynn and N. D'Angelo, Rev. Sci. Instr. 31, 1326 (1960).

13. In making the quantitative comparison between theory and experiment, we have used $\omega_{0}$, the measured damping at the highest magnetic field or the shortest column length for which $\omega_{g}$ is small, as the starting point. It is also necessary to assume $k_{z}=\frac{\pi}{2 L}$.

14. We are grateful to $B$. Coppi for pointing this out. 


\section{Figure Captions}

1. The signal is applied by a tone burst generator to a floating $2 \mathrm{~cm} \times 2 \mathrm{~cm}$ grid placed at the density gradient maximum and the wave is received by a Langmuir probe also placed at the density gradient maximum but axially displaced.

2a. Damping rate $\left(\omega_{i}=\omega_{0}-\omega_{g}\right)$ as a function of axial magnetic field. The density is $7 \times 10^{10}$, temperature is $2250^{\circ} \mathrm{K}$, and column length is $100 \mathrm{~cm}$. The excitation frequency $(10-20 \mathrm{KC})$ varies approximately as $\mathrm{B}^{-1}$ and is dependent upon the end plate temperature and its gradient.

$2 \mathrm{~b}$. Damping rate as a function of column length. The density is $6 \times 10^{10} / \mathrm{cc}$, temperature is $2300^{\circ} \mathrm{K}$, magnetic field is 1700 gauss. 


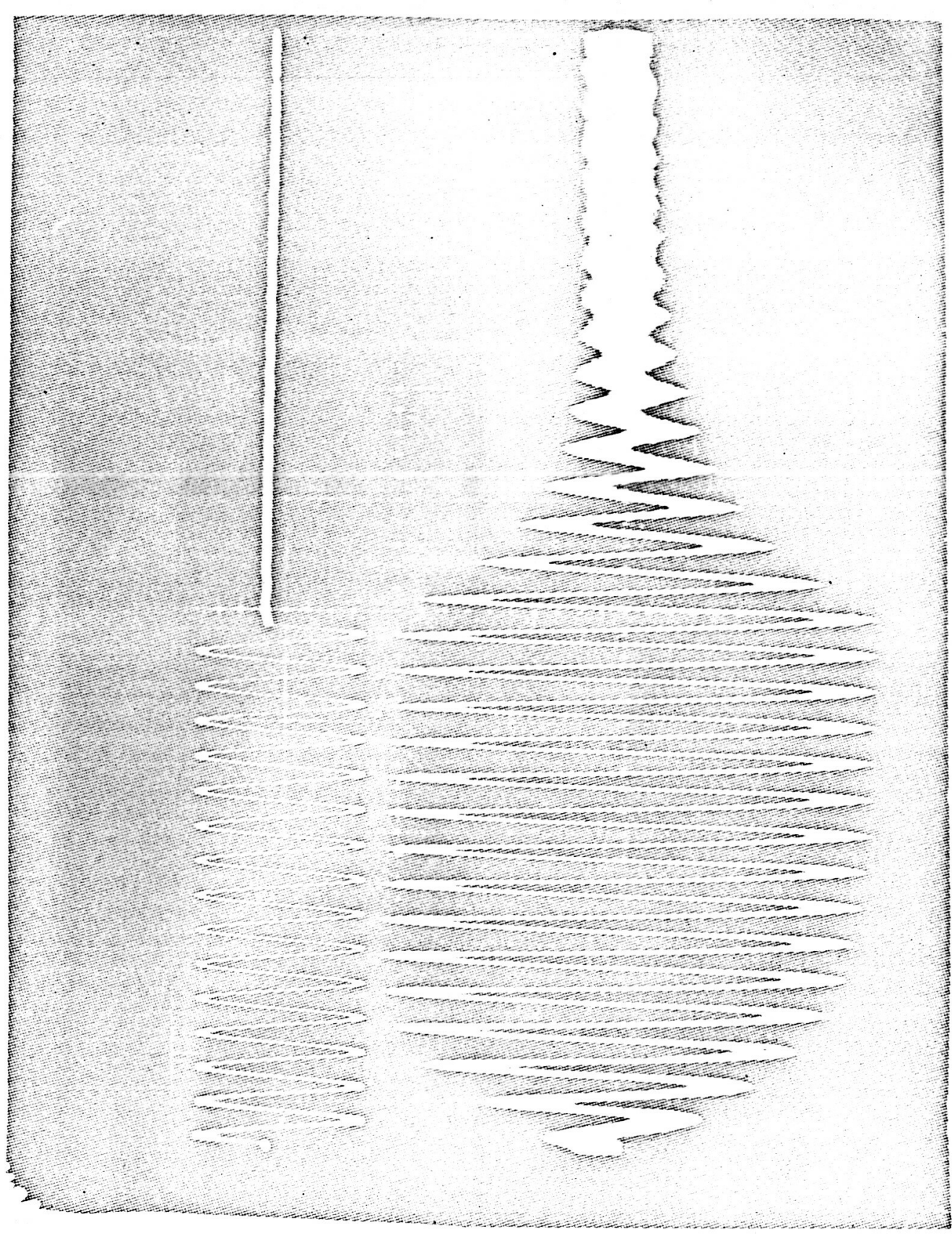

3
0
0
0
0
0
0
0
0
0
0
0

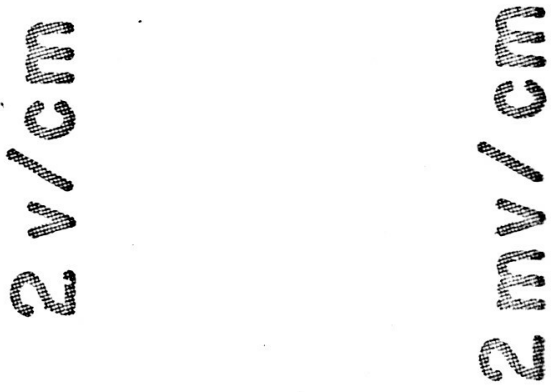




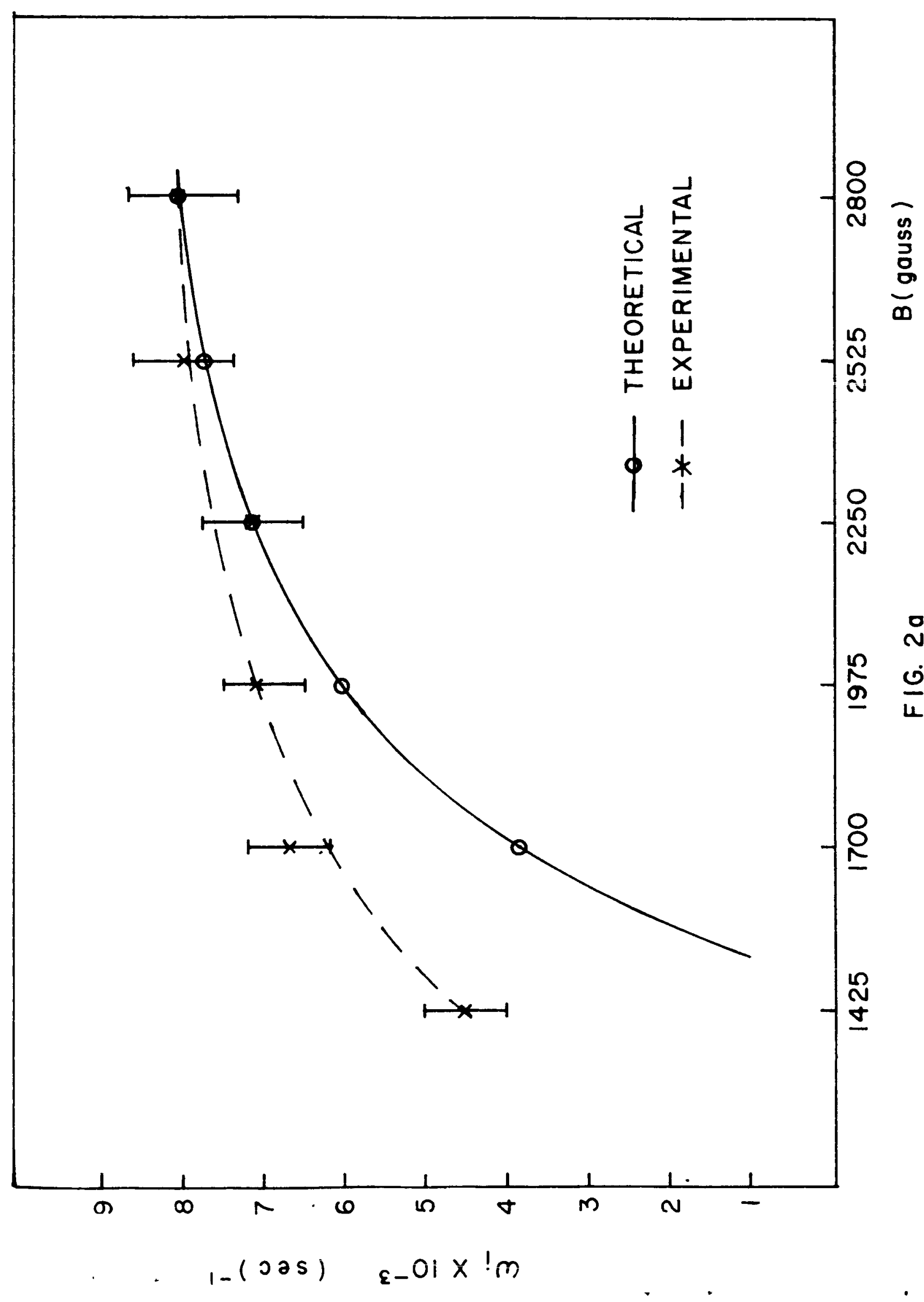




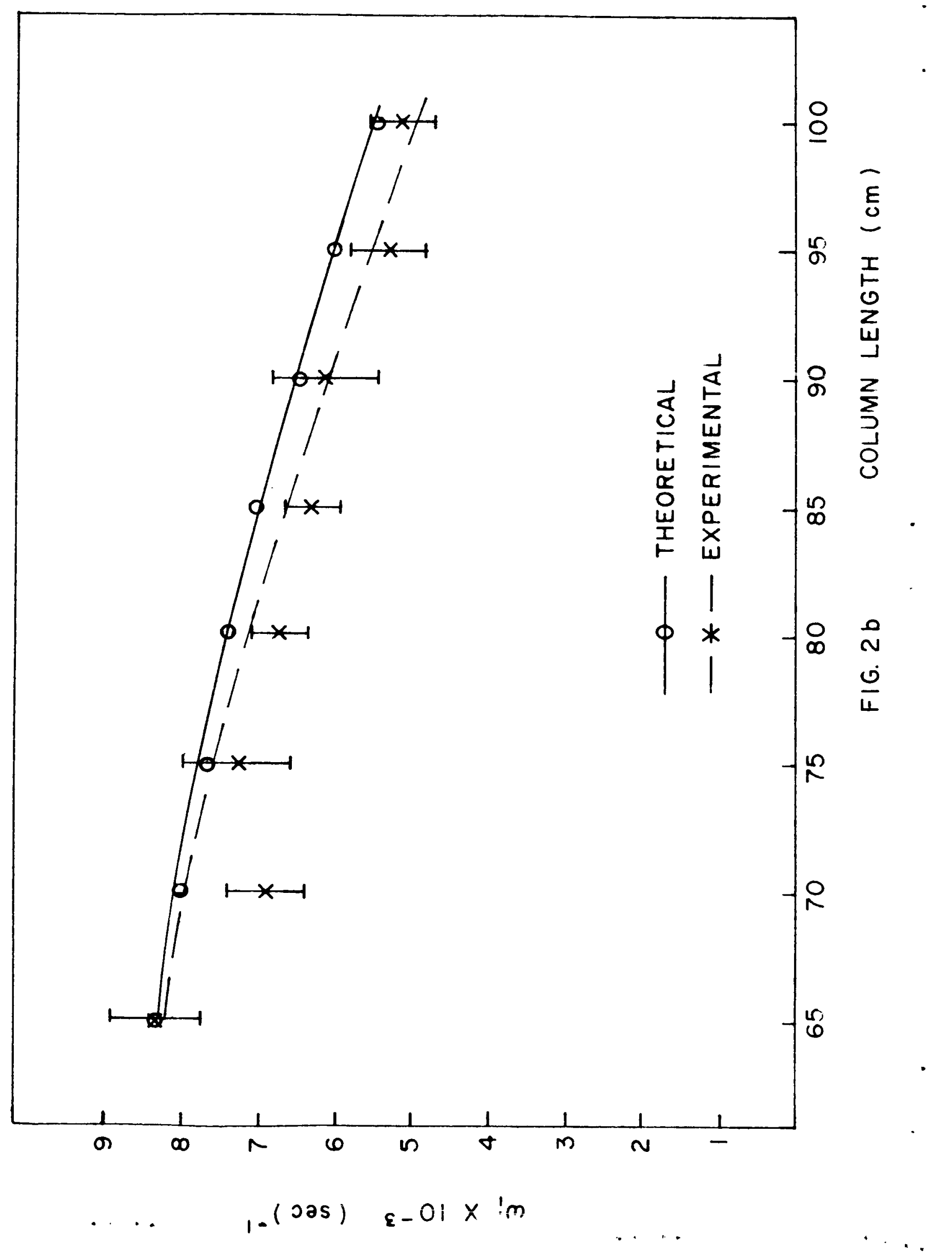

